\title{
EL DESARROLLO DE LAS CARACTERÍSTICAS MECÁNICAS DEL HORMIGÓN FABRICADO CON ADICIONES DE CENIZAS VOLANTES BAJO VARIAS CONDICIONES DE CURADO
}

\author{
(DEVELOPMENT OF MECHANICAL CHARACTERISTICS OF THE \\ CONCRETE MADE WITH FLY ASHES IN DIFFERENT CURING CONDITIONS)
}

Munther Latif Abdul Hussain, ingeniero civil IRAK

\author{
Directores de Tesis: \\ Juan José Moragues Terrades, catedrático de \\ "Edificación y Prefabricación". \\ Joaquín Catalá Alís, profesor titular de \\ "Proyectos" \\ Departamento de Ingeniería de la Construcción de la \\ Universidad Politécnica de Valencia-España
}

Fecha de recepción: 26 - IV - 93

\section{RESUMEN}

En esta investigación se analiza, experimentalmente, el desarrollo de las caracteristicas mecánicas de hormigones fabricados con diferentes porcentajes de sustitución del cemento portland por cenizas volantes y sometidos a la influencia de diversas condiciones de curado.

Muchas veces el conocimiento de la evolución de la resistencia a compresión del hormigón no es suficiente para detectar y resolver muestros problemas. Conocer el desarrollo de otras caracteristicas mecánicas del hormigón, especialmente a edades tempranas, es fundamental para establecer con seguridad el plazo de construcción así como el proceso constructivo. Nos referimos a la resistencia a tracción, módulo de elasticidad y adherencia.

Además, especialmente a edades tempranas, las condiciones de curado tienen una influencia importante en la evolución de las mencionadas características.

Por otro lado la sustitución parcial del cemento por cenizas volantes ticne un efecto adicional sobre aquélla.

En esta investigación se han analizado estos parámetros ensayando diferentes tipos de hormigones, con sustituciones de cemento por cenizas (clase F-ASTM) de un 20, 35 y 50 por cien, en peso; curados a temperaturas y humedades variando entre $10^{\circ} \mathrm{C}, 20^{\circ} \mathrm{C}$ y $30^{\circ} \mathrm{C}$ con el 60 y $100 \%$, respectivamente, realizándose los ensayos a 7,28 y 90 días, correspondientes a las cuatro caracteristicas mencionadas.

Se presentan en el trabajo tablas y gráficos de los resultados obtenidos, útiles para el diseño de procesos constructivos, con conclusiones cuantificadas de las variaciones obtenidas de estas características.

\section{SUMMARY}

In this research, we have made an experimental analysis of the development of the mechanical characteristics of concretes made with different percentages of fly ashes substituting Portland Cement and in different curing conditions.

It is often not enough to know the cyolution of the compressive strength in order to detect and solve our problems. It is very important to knou the development of other mechanical properties of concrete, especially at early stages, in order to determine with certainty the time the construction will take, as well as the construction process, i.e. tensile strength, Young's modulus and bond strength.

Furthermore, at early stages, the curing conditions are very important for the development of these mechanical properties.

Besides, the partial replacement of the cement by fly ashes has an additional influence on that development.

We have studied these parameters by testing different types of concretes, with 20,35 and 50 per cent $F$ class (ASTM) fly ashes replacing cement, cured at $10^{\circ} \mathrm{C}, 20^{\circ} \mathrm{C}$ and $30^{\circ} \mathrm{C}$ with 60 and 100 per cent relative humidity. The test stages were 7,28 and 90 days and all four properties were measured.

This paper presents various tables and figures with the test results which can be of use in the design of construction processes and some additional conclusions on the developments obtained.

Tesis leída el 9 de abril de 1992 en el Departamento de Ingeniería de la Construcción de la Universidad Politécnica de Valencia. Obtuvo la calificación de APTO CUM LAUDE. 


\section{Objetivo}

Esta tesis ha tenido como objetivo fundamental el análisis del desarrollo de las características resistentes de hormigones confeccionados con cenizas volantes como sustituto parcial del cemento en porcentajes de hasta un 50\%, sometidos a condiciones de curado variables, entre 10 y $40^{\circ} \mathrm{C}$ y humedades del 60 y $100 \%$.

Con ello se pretende pues:

- Conocer mejor el comportamiento de dichos hormigones con elevadas proporciones de C.V.

- Conocer el comportamiento de hormigones con "esas" C.V. en concreto, con sus características y procedencia.

- Conocer dicho comportamiento, además, para condiciones de curado ambientales lo más reales posible, aspecto fundamental para el análisis de ciertos procesos de descimbrado y desencofrado a corto plazo en que dichas condiciones difieren a lo largo del tiempo y desencofrado entre sí y con las de curado estándar.

\section{Planificación experimental. caracterización de los materiales}

Se ensayaron cuatro tipos de hormigón en función del porcentaje de sustitución (en peso) del cemento por C.V.: 0, 20, 35 y 50\%, sirviendo el primero de ellos de referencia y manteniéndose constante la cantidad de material cementante (cemento+C.V.).

Las edades a las que se realizaron los ensayos fueron: 7, 28 y 90 días.

Las dosificaciones se obtuvieron tras diversos ensayos previos. La del hormigón de referencia se analizó de modo que se obtuvieran resistencias a 28 días a compresión, de $20 \mathrm{MPa}$, con una consistencia del orden de 10 a $12 \mathrm{~cm}$. Dicha consistencia se ha procurado mantenerla constante, como requisito para su aplicación práctica en idénticas condiciones para los hormigones con C.V. En la tabla 1 se dan dichas dosificaciones tipo.

Las condiciones de curado simuladas en laboratorio fueron las que se adjuntan en la tabla 2.
Tabla 1.- Dosificaciones tipo

\begin{tabular}{|c|c|c|c|c|c|c|}
\cline { 2 - 6 } \multicolumn{1}{c|}{} & Arena & Grava & $\begin{array}{c}\text { Cemento } \\
(\mathrm{C})\end{array}$ & $\begin{array}{c}\text { Ceniza } \\
\text { Volante } \\
(\mathrm{F})\end{array}$ & $\begin{array}{c}\text { Agua } \\
(\mathrm{W})\end{array}$ & $\mathrm{W} /(\mathrm{C}+\mathrm{F})$ \\
\hline $\begin{array}{c}\text { De } \\
\text { referencia }\end{array}$ & $1.072,2$ & 789,74 & 290 & - & 215 & 0.74 \\
\hline $\begin{array}{c}\text { F.A.C. } \\
\text { (con cenizas) }\end{array}$ & $1.076,65$ & 794,52 & 145 & 145 & 197.5 & 0.68 \\
\hline
\end{tabular}

Tabla 2.- Condiciones de curado inicial

\begin{tabular}{|c|c|c|}
\hline Curado № & Temperatura $^{\circ} \mathrm{C}$ & Humedad $\%$ \\
\hline 1 & 20 & 60 \\
2 & 30 & 60 \\
3 & 40 & 60 \\
4 & 10 & 100 \\
5 & 20 & 100 \\
6 & 30 & 100 \\
\hline
\end{tabular}

Tabla 3.- Características del cemento y de las cenizas volantes

\begin{tabular}{|c|c|c|}
\hline & Cenizas volantes & Cemento \\
\hline $\mathrm{SiO}_{2}$ & $43,31 \%$ & $20,07 \%$ \\
\hline $\mathrm{SO}_{3}$ & $1,31 \%$ & $3,31 \%$ \\
\hline $\mathrm{CaO}$ & $5,71 \%$ & $60,15 \%$ \\
\hline CaO libre & $0,2 \%$ & $1,86 \%$ \\
\hline $\mathrm{MgO}$ & $1,62 \%$ & $1,89 \%$ \\
\hline $\mathrm{Fe}_{2} \mathrm{O}_{3}$ & $18.35 \%$ & $7,29 \%$ \\
\hline $\mathrm{Al}_{2} \mathrm{O}_{3}$ & $28,05 \%$ & $2,61 \%$ \\
\hline $\mathrm{Na}_{2} \mathrm{O}$ & $0,14 \%$ & $0.19 \%$ \\
\hline $\mathrm{K}_{2} \mathrm{O}$ & $0.55 \%$ & $0,71 \%$ \\
\hline Humedad & $0,32 \%$ & $0,27 \%$ \\
\hline Pérdida ignición & $0,85 \%$ & $2,46 \%$ \\
\hline R.I. (Residuo insoluble) & $1,40 \%$ & $1,42 \%$ \\
\hline
\end{tabular}

Los parámetros resistentes ensayados fueron:

- Resistencia a compresión simple, mediante ensayos sobre probetas cilindricas ( $\varnothing 15 \times 30)$, según la Norma UNE 83-304-84.

- Módulo de elasticidad, aplicando la Norma ASTM C-469 a probetas similares a lasanteriores.

- Resistencia a tracción, aplicando el "ensayo brasileño" según la Norma UNE 83-306-85. 
- Adherencia (media y última) mediante el ensayo "pull-out" modificado, según la Norma RILEM RC-6, con barras de $16 \mathrm{~mm}$.

Tanto los cementos, como los áridos y las C.V. fueron sometidos a los correspondientes ensayos de recepción. Las características de las C.V. se dan en la tabla 3. Corresponden a la clase F (ASTM) y proceden de la Central Térmica de ANDORRA (Teruel).

La confección del hormigón, así como los ensayos de recepción, los previos y los del hormigón se planificaron y realizaron en el Laboratorio de Materiales del Departamento de Ingeniería de la Construcción de la Universidad Politécnica de Valencia.

\section{Resultados}

Los resultados experimentales, agrupados para cada una de las características resistentes indicadas, tras presentarse los valores directamente deducidos de los ensayos, se agrupan para su análisis en forma de gráficos y tablas.

Los gráficos, en número de 60, corresponden a dos tipos:

a) En el primero de ellos se da la variación de los parámetros (5) resistentes (6 gráficos para cada uno de ellos) en función del porcentaje de cenizas volantes, con una línea para cada valor de la temperatura (los 6 gráficos diferentes corresponden a la variación de la humedad 60 y $100 \%$ y de la edad 7, 28 y 90 días). Se adjuntan como ejemplo los tres gráficos correspondientes a la resistencia a tracción $(7,28$ y 90 días) para una humedad relativa del 60\% (figuras 1 a 3 ).

b) En el segundo tipo se da la variación de la relación entre dichos parámetros resistentes y el correspondiente a 28 días en curado estándar, en función de la edad del hormigón, con una línea para cada porcentaje de cenizas (los 6 gráficos diferentes para cada parámetro resistente corresponden a la variación de la humedad -60 y $100 \%$ y de la temperatura- 20 , 30 y $40^{\circ} \mathrm{C}$ para el $60 \%$ de H.R. y 10,20 y $30^{\circ} \mathrm{C}$ para el $100 \%$ de H.R.). Se adjuntan también, como ejemplo, tres gráficos correspondientes en este caso a la resistencia a compresión, para la humedad del 100\% (figuras 4 a 6).
Humedad relativa $60 \%$ (edad 7 dias)

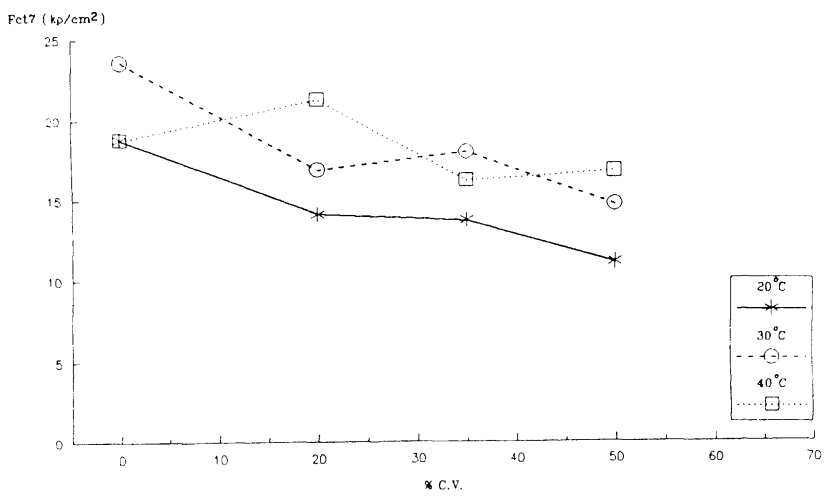

Fig 1.- Resistencia tracción a 7 dias (Fct7) para diferentes porcentajes de C.V. en función de la temperatura.

Humedad relativa $60 \%$ (edad 28 días)

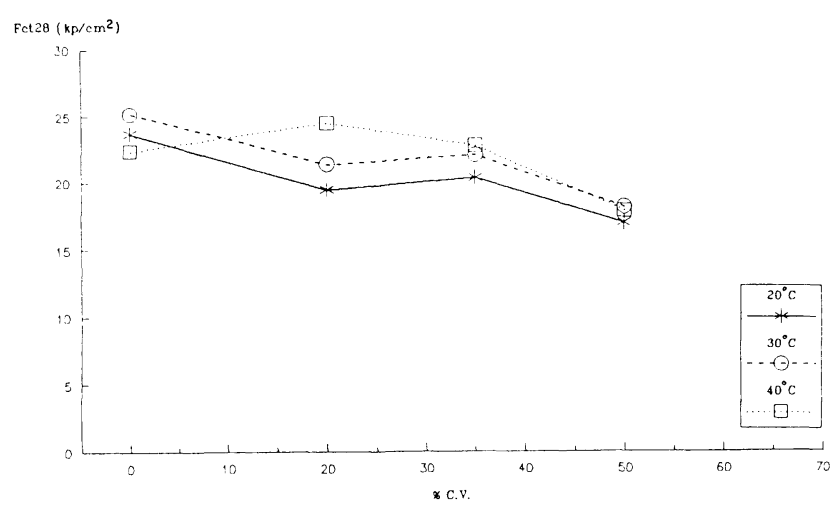

Fig 2.- Resistencia tración a 28 dias (Fot28) para diferentes porcentajes de C.V. en función de la temperatura.

Humedad relativa 60\% (edad 90 días)

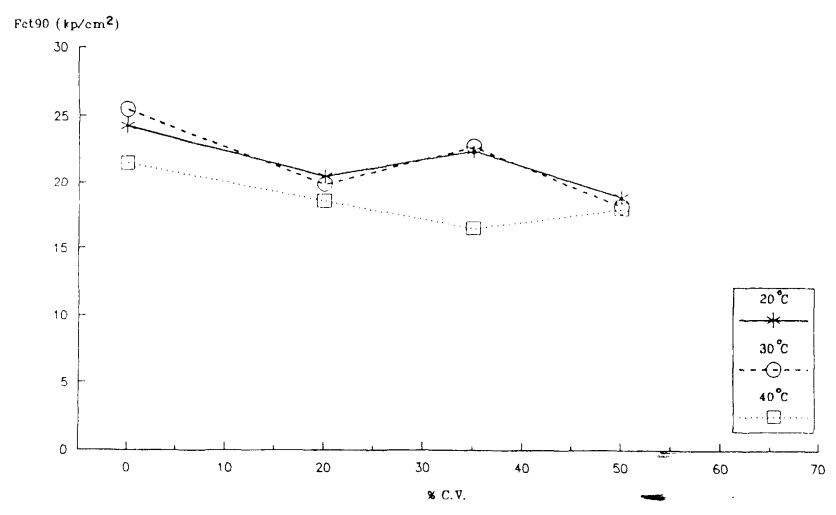

Fig 3. - Resistencia tración a 90 dias (Fotgo) para diferentes porcentajes de C.V. en función de la temperatura. 
Condición de curado (T.: $10^{\circ} \mathrm{C}$, H.R.: $100 \%$ )

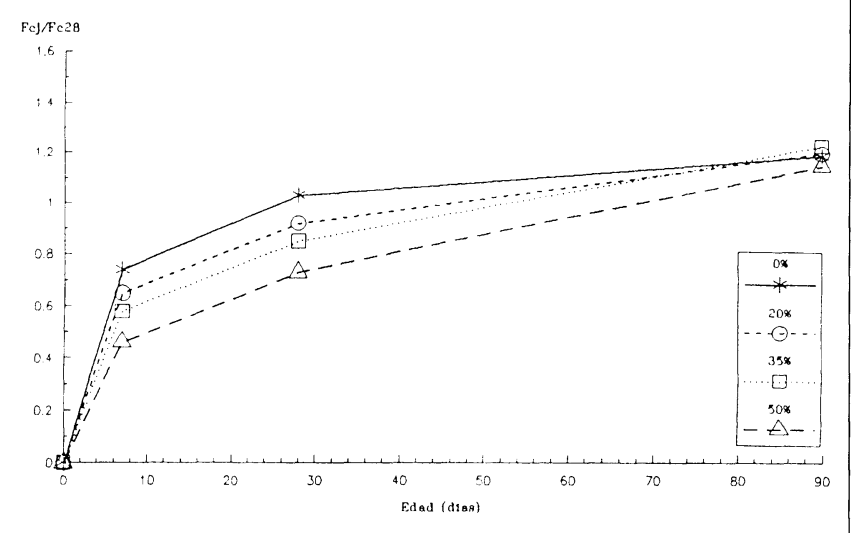

Fig 4.- Resistencia a compresión en función de la resistencia a compresión a 28 dias en curado estándar para diferentes porcentajes de C.V.

\section{Condición de curado (T.: $20^{\circ}$ C, H.R.: $100 \%$ )}

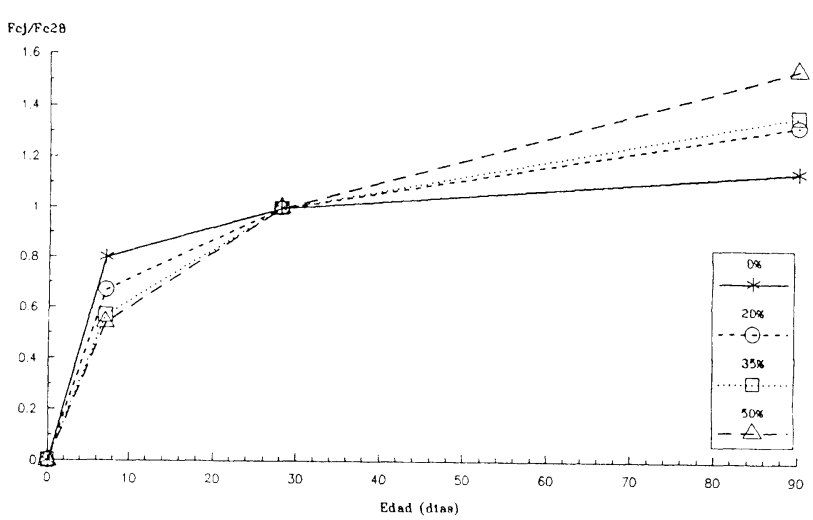

Fig 5.- Resistencia a compresión en función de la resistencia a compresión a 28 días en curado estándar para diferentes porcentajes de C.V.

\section{Condición de curado (T.: $30^{\circ} \mathrm{C}$, H.R.: $100 \%$ )}

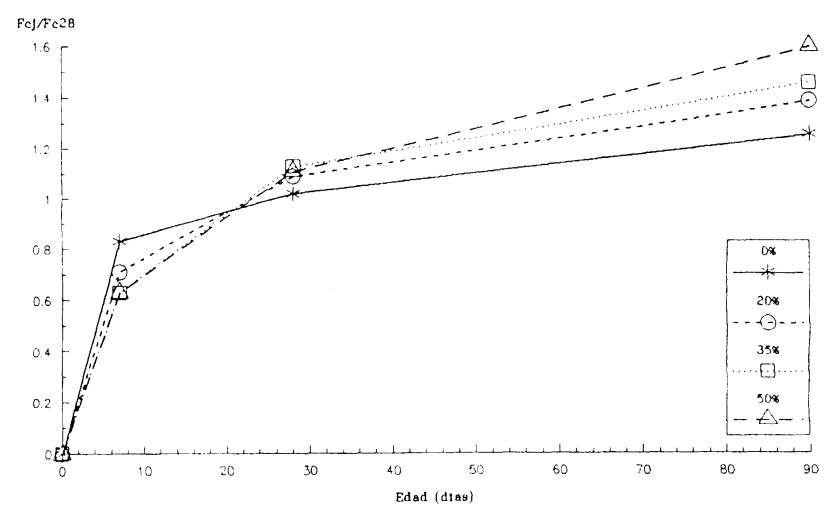

Fig 6.- Resistencia a compresión en función de la resistencia a compresión a 28 dias en curado estandar para diferentes porcentajes de C.V.
Tabla 4

\begin{tabular}{|c|c|c|c|}
\hline \multicolumn{4}{|c|}{ Condición de curado } \\
\hline & $=20^{\circ} \mathrm{C}$ & $H \cdot R .=60^{\circ}$ & \\
\hline \multirow{2}{*}{$\%$ C.V. } & \multicolumn{3}{|c|}{ Resistencia a compresión } \\
\hline & 7 dias & 28 días & 90 días \\
\hline $\begin{array}{c}\text { Hormigón } \\
\text { Referencia } \\
0 \%\end{array}$ & 100 & 100 & 100 \\
\hline $\begin{array}{l}\text { Hormigón } \\
\text { con } 20 \%\end{array}$ & 75 & 79 & 92 \\
\hline $\begin{array}{l}\text { Hormigón } \\
\text { con 35\% }\end{array}$ & 80 & 95 & 104 \\
\hline $\begin{array}{l}\text { Hormigón } \\
\text { con } 50 \%\end{array}$ & 65 & 76 & 85 \\
\hline
\end{tabular}

\section{Tabla 5}

\begin{tabular}{|c|c|c|c|}
\hline \multicolumn{4}{|c|}{ Condición de curado } \\
$\mathrm{T} .=20^{\circ} \mathrm{C}$ & \multicolumn{3}{|c|}{ H.R. $60 \%$} \\
\hline \multirow{2}{*}{$\%$ C.V. } & \multicolumn{2}{|c|}{ Resistencia a compresión } \\
\cline { 2 - 4 } & 7 dias & 28 dias & $\mathbf{9 0}$ dias \\
\hline $\begin{array}{l}\text { Hormigón } \\
\text { Referencia } \\
\mathbf{0} \%\end{array}$ & 82 & 100 & 104 \\
\hline $\begin{array}{l}\text { Hormigón } \\
\text { con 20\% }\end{array}$ & 77 & 100 & 121 \\
\hline $\begin{array}{l}\text { Hormigón } \\
\text { con 35\% }\end{array}$ & 69 & 100 & 114 \\
\hline $\begin{array}{l}\text { Hormigón } \\
\text { con 50\% }\end{array}$ & 69 & 100 & 116 \\
\hline
\end{tabular}

En las conclusiones se dan además otras 100 tablas agrupadas en cuatro casos:

Caso a: Valores obtenidos, en porcentaje, con respecto al hormigón de referencia (sin cenizas), para cada parámetro resistente y cada condición de curado (30). Véase tabla 4 para $20^{\circ} \mathrm{C}$ y $60 \%$ y resistencia a compresión.

Caso b: Idem, con respecto al hormigón a 28 días correspondiente (30). Véase tabla 5 para $20^{\circ} \mathrm{C}$ y $60 \%$ y resistencia a compresión también.

Caso c: Idem, con respecto al hormigón en condiciones de curado estándar para cada edad; para cada porcentaje de cenizas $¥$ parámetro resistente (20). Véase tabla 6 para $35 \%$ de cenizas (C.V.), resistencia a compresión. 
Tabla 6 (35\% de C.V.)

\begin{tabular}{|c|c|c|c|c|}
\hline \multicolumn{2}{|c|}{ Condición de curado } & \multicolumn{3}{|c|}{ Resistencia a compresión } \\
\hline T. ${ }^{\circ} \mathbf{C}$ & H.R. $\%$ & 7 dias & $\mathbf{2 8}$ dias & $\mathbf{9 0}$ días \\
\hline $\mathbf{1 0}$ & $\mathbf{1 0 0}$ & 103 & 86 & 90 \\
\hline \multirow{2}{*}{$\mathbf{2 0}$} & $\mathbf{6 0}$ & 132 & 108 & 89 \\
\cline { 2 - 5 } & $\mathbf{1 0 0}$ & 100 & 100 & 100 \\
\hline \multirow{2}{*}{30} & $\mathbf{6 0}$ & 169 & 137 & 109 \\
\cline { 2 - 5 } & $\mathbf{1 0 0}$ & 122 & 124 & 117 \\
\hline \multirow{2}{*}{$\mathbf{4 0}$} & $\mathbf{6 0}$ & 166 & 127 & 92 \\
\hline
\end{tabular}

Tabla 7 (35\% de C.V.)

\begin{tabular}{|c|c|c|c|c|c|}
\hline \multicolumn{2}{|c|}{ Condición de curado } & \multicolumn{4}{|c|}{ Resistencia a compresión } \\
\hline \multirow{2}{*}{ T. ${ }^{\circ}$ C } & H.R. \% & 7 dias & $\mathbf{2 8}$ dias & $\mathbf{9 0}$ dias & $\begin{array}{c}\text { 28 dias } \\
\text { Referencia }\end{array}$ \\
\hline $\mathbf{1 0}$ & $\mathbf{1 0 0}$ & 58 & 85 & 123 & 100 \\
\hline \multirow{2}{*}{$\mathbf{2 0}$} & $\mathbf{6 0}$ & 69 & 98 & 112 & 100 \\
\cline { 2 - 6 } & $\mathbf{1 0 0}$ & 57 & 100 & 137 & 100 \\
\hline \multirow{2}{*}{$\mathbf{3 0}$} & $\mathbf{6 0}$ & 70 & 101 & 111 & 100 \\
\cline { 2 - 6 } & $\mathbf{1 0 0}$ & 63 & 113 & 146 & 100 \\
\hline \multirow{2}{*}{$\mathbf{4 0}$} & $\mathbf{6 0}$ & 77 & 104 & 104 & 100 \\
\hline
\end{tabular}

Caso $d$ : Idem, con respecto a los valores obtenidos a 28 días bajo curado estándar, para cada porcentaje de cenizas y cada parámetro resistente (20). Véase tabla 7 para $35 \%$ de C.V. y resistencia a compresión.

\section{Conclusiones}

Podemos entresacar las siguientes:

Las tablas pueden servir de múltiples usos, tanto para seleccionar porcentajes de cenizas como para ver efectos del curado y, sobre todo, para tener valores relativos que permitan elecciones de C.V. adecuadas a las necesidades particulares de la obra.

El efecto lubricante de las cenizas es evidente, pues al adicionar el 50\% de C.V. ha sido necesario reducir la cantidad de agua para mantener la consistencia.
Por otro lado se observa, a nivel general, un cambio apreciable al pasar de adiciones del 35\% al $50 \%$ en las características de los hormigones. Parece que situar como porcentaje inferior de definición de hormigones con alto contenido en C.V. el 35\% es bastante adecuado.

Se ha confirmado el lento desarrollo de la resistencia a compresión de hormigones con C.V. Así, en condiciones de curado estándar, a los 7 días tenemos déficit entre el 14 y $60 \%$, y a los 28 días del 20 al 50\%, siempre con respecto al hormigón de referencia y con porcentajes de cenizas del 20 al 50\%. A 90 días, los hormigones con $20 \%$ de cenizas alcanzan la misma resistencia que el hormigón de referencia, existiendo todavía pérdidas de hasta el 30\% si tenemos un 50\% de cenizas. En cualquier caso vemos una importante evolución de la resistencia a compresión entre 28 y 90 días, con incremento del 33 al 55\%, mayores a mayor cantidad de cenizas.

Vemos pues que la edad de 90 días puede ser una buena referencia para hormigones con bajo contenido en C.V. $(\leq 35 \%)$, pero queda corta en el caso de mayores cantidades de cenizas.

La resistencia a compresión es muy sensible a las condiciones de curado de estos hormigones, aumentando esta sensibilidad con la cantidad de cenizas adicionadas. Se han podido constatar los perniciosos efectos de la falta de humedad sobre los valores que se alcanzan de la resistencia a compresión, a cualquier edad. Sin embargo, un aumento de la temperatura es beneficioso, especialmente a corto plazo.

La adición de las C.V. al hormigón influye menos sobre su módulo de elasticidad que sobre la resistencia a compresión de modo que, en condiciones de curado estándar, a 7 y 28 días se pierde entre el 15 y $20 \%$ de la rigidez con respecto a un hormigón normal, recuperándose con creces a $\operatorname{los} 90$ días.

Aunque sin alcanzar la gran sensibilidad a las condiciones de curado que presenta la resistencia a compresión de hormigones con C.V., el módulo de deformación también viene afectado por ellas, tanto más cuanto mayor porcentaje de cenizas dispongamos. Así, curados secos reducen, incluso a largo plazo, el módulo de deformación entre un 17 y un $27 \%$ con respecto al que tendríamos con curado estándar. Nuevamente se observa que temperaturas elevadas producen mejoras de rigidez 
a los 7 días, mientras que las bajas temperaturas afectan menos al módulo de deformación que a la resistencia a compresión.

La resistencia a tracción evoluciona algo más rápidamente que la resistencia a compresión a edades tempranas cuando adicionamos C.V. Pero posteriormente se consiguen escasas mejoras de la resistencia a tracción con el tiempo. Así, de 28 a 90 días, en el mejor de los casos, sólo hay mejoras del $15 \%$, siempre algo mayores que las de un hormigón sin cenizas. Observamos pues una gran disparidad con respecto a lo que ocurría con la resistencia a compresión.

Las altas temperaturas benefician la resistencia a tracción de hormigones con C.V. a edades jóvenes. Curados fríos y secos afectan mucho a la resistencia a tracción de estos hormigones. Es curioso constatar que el curado estándar es el que ofrece siempre mejores valores de la resistencia a tracción a largo plazo, independientemente de que el hormigón tenga o no C.V. Esto no hace más que abundar en el diferente comportamiento entre la resistencia a compresión y a tracción al variar las condiciones de curado y adicionar C.V.
Las C.V. afectan de modo semejante a la tensión media y a la tensión última de adherencia. En condiciones de curado estándar la adherencia de estos hormigones se comporta de modo semejante a como lo hacía la resistencia a compresión. Así, a 7 días se producen pérdidas del 23 al 55\% con respecto al hormigón de referencia para hormigones con el 20 y el 50\% de C.V., respectivamente. A los 90 días, hormigones con bajos contenidos de C.V. alcanzan adherencias semejantes al hormigón de control. No ocurre así en hormigones con alto contenido de C.V. que a 90 días tienen déficits de adherencia del orden del $15 \%$. Dada la gran evolución de la adherencia con el tiempo en hormigones con cenizas, la edad de 90 días vuelve a parecer escasa como referencia para estos hormigones.

Nuevamente las condiciones de curado afectan más cuanto mayor es el porcentaje de cenizas en el hormigón. Así, curados fríos afectan muy negativamente a la adherencia. Las altas temperaturas mejoran ligeramente la adherencia a edades jóvenes pero, a largo plazo, son perniciosas. Vuelven a ser las condiciones de curado estándar las que, generalmente, proporcionan mejores valores de la adherencia a largo plazo.

\section{ALOJAMIENTO Y TECNOLOGÍA: ¿INDUSTRIALIZACIÓN ABIERTA? Julián Salas Serrano}

La experiencia nacional en construcción industrializada en la última década, aunque no abundante, puede resultar paralizante Como reacción, este trabajo trata de elaborar y ordenar la información que, pegado al terreno, se ha acumulado durante los sesenta.

Auscultando tendencias avanzadas en otros paises y apoyándose en nuestra realidad cotidiana, el autor de este trabajo apuesta por la industrialización, presintiendo un futuro con pocos puntos en común con lo que en general, hasta bien reciente, se ha conocido como construcción industrializada.

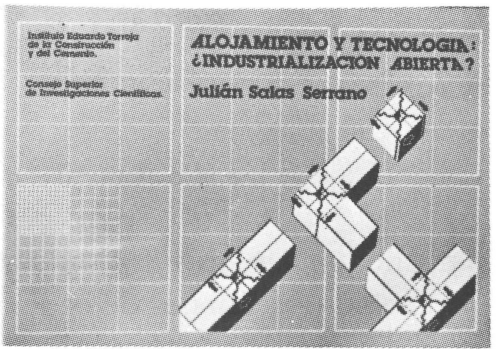

Se abre el trabajo con un prólogo del Profesor Ciribini en el cual, con rigor conceptual y desde su rica experiencia, analiza algunos de los conceptos más polémicos del fenómen de industrialización del sector construcción. El autor recopila un conjunto de aportaciones de maestros de la arquitectura al lento proceso de evolución tecnológica y conceptual continuando con un intento de respuesta realista a la interrogante que flota en el sector nacional: ¿réquiem por la industrialización?

Se dedican otros capitulos a revisar lo realizado y a encarar el futuro inmediato: el concepto de componente, su repercusión económica, la dificultad del proyecto a base de productos industriales, la nueva vigencia embrionaria de la construcción por medio de catálogos, los conceptos de flexibilidad e intercambiabilidad desde una óptica práctica... Especial atención dedica este trabajo a la exposición de lo que podíamos llamar nuevos derroteros de la coordinación dimensional, aportando una visión actualizada y pragmática de los enfoques más implantados en Europa.

Las siempre problemáticas interrelaciones entre normativa, calidad e industria se detallan de forma documentada y realista. Finaliza el trabajo con unas reflexiones dirigidas a ámbito empresarial y un esbozo de "reglas de juego» que faciliten al subsector la salida del «impasse» actual.

Un volumen encuadernado en rústica, de $24 \times 16,5 \mathrm{~cm}$, compuesto de 160 páginas, con 109 figuras, 19 tablas y 86 referencias bibliográficas. 